\title{
EVOLUÇÃO DO ESCORREGAMENTO EPIFISÁRIO PROXIMAL DO FÊMUR APÓS TRATAMENTO NÃO CIRÚRGICO
}

\section{EVOLUTION OF SLIPPED CAPITAL FEMORAL EPIPHYSIS AFTER NONSURGICAL TREATMENT}

\author{
Cláudio Santili' ${ }^{1}$, Miguel Akkari², Gilberto Waisberg ${ }^{3}$, Susana Reis Braga ${ }^{4}$, Akemi Kasahara $^{5}$, Mauro Coura Perez $^{5}$
}

\section{RESUMO}

Objetivo: Avaliar a eficácia do tratamento conservador em pacientes com epifisiólise proximal do fêmur (EEPF) e as complicações devidas à evolução da doença. Métodos: Foram analisados, retrospectivamente, 18 pacientes (26 quadris) consecutivamente atendidos no período entre dezembro de 1996 e agosto de 2006 no Serviço de Ortopedia da Santa Casa de Misericórdia de São Paulo, encaminhados por outros serviços com diagnóstico de EEPF e tratados de forma não cirúrgica. Resultados: A progressão do escorregamento aconteceu em 19 quadris (73\%), sendo que, dos quadris com escorregamento leve, oito permaneceram leves, quatro progrediram para moderados e um tornou-se grave pela classificação Southwick. Dos seis quadris classificados como moderados, quatro evoluíram para grave e os dois graves acentuaram-se um pouco mais. Conclusões: Apesar de a indicação cirúrgica ser hoje consenso no tratamento do EEEP para evitar a progressão do escorregamento, há ainda pacientes com diagnóstico confirmado que são tratados de forma conservadora, e isto representa um grande erro, pois implica no aumento da morbidade da doença.

Descritores - Epífise deslocada; Terapêutica; História natural da doença

\section{ABSTRACT}

Objective: To evaluate the efficacy of conservative treatment of slipped capital femoral epiphysis (SCFE) and the complications due to the progression of the slip. Methods: 18 patients (26 hips) seen consecutively from December 1996 to August 2006 at the Orthopedics Service of Santa Casa de Misericórdia de São Paulo, referred from other services with a SCFE diagnosis and treated without surgery were retrospectively analyzed. Results: Slip progression was observed in 19 hips (73\%). Among the mild cases, 8 remained mild, four turned moderate and one became severe according to Southwick classification. Four out of the six originally moderate cases became severe and the two already severe cases worsened. Conclusion: Despite there being a consensus regarding the use of surgical treatment to prevent SCFE progression, some cases are still being treated conservatively. This represents a grave error, since it increases the morbidity of the disease.

Keywords - Epiphyses, Slipped; Therapeutics; Natural history of diseases

\section{INTRODUÇÃO}

No escorregamento da epífise proximal do fêmur (EEPF) ocorre deslocamento gradual e progressivo da epífise em relação ao colo, processo esse resultante de forças de cisalhamento e de compressão sobre uma fise doente $^{(1)}$. Em grande parte dos pacientes, a doença acomete crianças com sobrepeso, ou mesmo obesas, no período da adolescência e pré-adolescência. Proporcionalmente maior risco de acometimento é constatado em

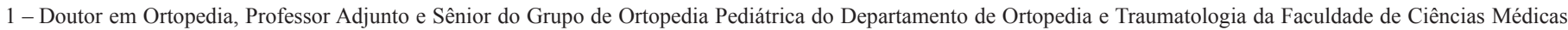
da Santa Casa de São Paulo.

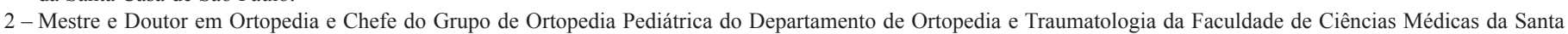
Casa de São Paulo.

3 - Médico Assistente do Grupo de Ortopedia Pediátrica do Departamento de Ortopedia e Traumatologia da Faculdade de Ciências Médicas da Santa Casa de São Paulo.

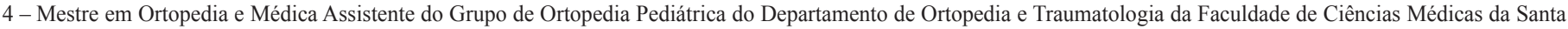
Casa de São Paulo.

5 - Médico estagiário do Grupo de Ortopedia Pediátrica do Departamento de Ortopedia e Traumatologia da Faculdade de Ciências Médicas da Santa Casa de São Paulo.

Trabalho realizado no Grupo de Ortopedia Pediátrica, Departamento de Ortopedia e Traumatologia da Faculdade de Ciências Médicas da Santa Casa de São Paulo Correspondência: Rua Mato Grosso, 306 Conjunto 1212 - 01239-040 - Higienopolis - São Paulo, SP.

E-mail: ortopedsantili@gmail.com 
pacientes que se encontram em reposição hormonal ou portadores de endocrinopatias como hipotiroidismo e hipogonadismo $^{(2)}$. Na avaliação clínica, a marcha claudicante associada à dor na virilha, que por vezes irradia-se para a face interna da coxa e/ou joelho, são sintomas muitas vezes menosprezados no primeiro atendimento, retardando o correto diagnóstico. Estes sintomas insidiosos induzem ao tratamento não definitivo da doença e fomentam a ideia errônea de que a utilização de sintomáticos, a retirada da carga, a tração do membro seguida de imobilização gessada ou até mesmo a simples observação ${ }^{(3)}$ seriam suficientes para interromper a evolução progressiva do escorregamento.

Carney et $a l^{(4)}$ demonstraram que $17 \%$ dos casos de EEPF progrediram com deslizamento adicional quando não tratados cirurgicamente. Ordeberg et $a l^{(5)}$ comprovaram que a permanência da fise aberta caracteriza-se como risco de progressão para o deslizamento após o diagnóstico.

Este trabalho tem por objetivo avaliar a eficácia do tratamento conservador em pacientes com EEPF e as complicações devidas à evolução da doença.

\section{MÉTODOS}

Este é um estudo observacional retrospectivo baseado na avaliação de pacientes atendidos no período compreendido entre dezembro de 1996 e agosto de 2006 com diagnóstico de EEPF no Grupo de Ortopedia Pediátrica da Santa Casa de Misericórdia de São Paulo. O projeto deste estudo foi submetido e aprovado pelo Comitê de Ética Médica da Instituição (protocolo 384/07).

Foram critérios de inclusão:

1) Pacientes previamente atendidos e diagnosticados com EEPF, tratados de forma não cirúrgica, com anti-inflamatórios não hormonais (AINEs), fisioterapia, repouso, muletas ou gesso, e que, posteriormente, por não sentirem melhora, procuraram voluntariamente o Serviço de Ortopedia Pediátrica da Santa Casa de São Paulo.

2) Existência de radiografias prévias à chegada no serviço que comprovassem que o diagnóstico de EEPF havia sido feito, de pacientes que tinham acompanhamento médico regular.

Foram critérios de exclusão:

1) Pacientes que não haviam sido diagnosticados como EEPF;

2) Pacientes que não possuíam documentação radiográfica;
3) Pacientes que haviam sido tratados da dor no quadril com outro diagnóstico (exemplo: contusão na coxa);

4) Pacientes que não aceitaram o tratamento cirúrgico proposto no diagnóstico inicial;

5) Pacientes que possuíam endocrinopatia concomitante;

6) Pacientes previamente diagnosticados, mas que foram encaminhados ao tratamento cirúrgico (e não ao conservador) no serviço.

Todos os prontuários dos pacientes incluídos foram analisados, e os dados demográficos e os referentes à história clínica relatada e à evolução do quadro mórbido foram registrados. Foram também registrados os períodos de tempo entre radiografias e entre radiografias e tratamentos.

A avaliação da progressão do deslizamento foi feita pela medição do ângulo epifísio-diafisário de Southwick $^{(6)}$. Considera-se como normal o ângulo epifísio-diafisário na posição de perfil de até $10^{\circ}$ (tanto para anterior como para posterior). Na classificação de Southwick, os graus de deslizamento são subdivididos de acordo com o ângulo, sendo definidos como leves quando o ângulo for de até $30^{\circ}$; moderados, se entre $30^{\circ}$ e $60^{\circ}$; e graves, quando maiores que $60^{\circ}$.

$\mathrm{Na}$ análise estatística, foi avaliada a progressão do deslizamento entre as radiografias inicial e pré-operatória imediata, e estes dados foram relacionados com a idade, com o período de tempo do escorregamento e o gênero acometido. Adotou-se o nível de 5\% $(0,005)$ para aplicação dos testes de significância. Na análise das correlações, aplicaram-se os testes de Mann-Whitney, análise de correlação de Spearman e teste dos postos sinalizados de Wilcoxon. Utilizou-se o programa SPSS (Statistical Package for Social Sciences), em sua versão 13.0, para a obtenção dos resultados.

\section{RESULTADOS}

No período do estudo, foram admitidos 401 pacientes com epifisiólise de diversos graus. Entre os pacientes atendidos, 35 (8,7\%) pacientes com diagnóstico prévio de EEFE foram submetidos a tentativa de tratamento conservador prévio ao cirúrgico. Porém, usando os critérios de inclusão e exclusão deste estudo, restaram 18 (4,5\%) pacientes.

Entre os 18 pacientes, três quadris $(16,7 \%)$ eram acometidos do lado esquerdo, sete $(38,9 \%)$ à direita e oito $(44,4 \%)$ bilateralmente, perfazendo um total de 26 quadris. A idade dos pacientes variou de nove anos e 10 meses a 15 anos e oito meses, com média de 12 anos e 
sete meses, sendo 10 adolescentes do sexo masculino e oito do sexo feminino.

Através da análise retrospectiva das informações contidas nos prontuários, observamos que os pacientes procuraram o serviço devido à persistência da queixa álgica e progressão da limitação funcional, apesar do tratamento conservador instituído. O período entre as radiografias iniciais e as pré-operatórias, produzidas no nosso serviço, variou desde 26 até 402 dias.

Todos os 18 pacientes incluídos no estudo tinham como queixa a dor refratária ao tratamento conservador instituído previamente, motivo pelo qual procuraram o serviço. Na radiografia inicial dos 26 quadris, segundo a classificação de Southwick, observou-se que $18(69,2 \%)$ foram classificados como escorregamentos leves, seis $(23 \%)$ moderados e dois $(7,8 \%)$, graves. Dos 24 quadris inicialmente enquadrados como leves ou moderados na radiografia inicial, houve piora do escorregamento em nove (37,5\%) na comparação com a radiografia pré-operatória, segundo a classificação utilizada, sendo que entre os 18 casos leves, quatro $(16,6 \%)$ progrediram para moderado e um $(4,15 \%)$, para grave. Dos inicialmente moderados, quatro $(16,6 \%)$ evoluíram para grave. A análise demonstra a progressão do escorregamento (agravamento) em 19 (73\%) dos 26 quadris (Tabela 1 e Figura 1).

Na Tabela 2 está demonstrada a análise estatística dos dados obtidos e que foram avaliados de acordo com as variáveis: tempo, idade, sexo e grau de deslizamento. Na comparação entre ambos os sexos, para as variáveis tempo, idade e grau de deslizamento, observamos que o tempo $(p=0,03)$ e a idade $(p=0,03)$ apresentam diferenças estatisticamente significantes, quando comparamos ambos os sexos entre si, com as meninas acometidas com menor média de idade que os meninos, enquanto que o grau de deslizamento não foi diferente entre os sexos $(\mathrm{p}=0,890)$. O tempo $(\mathrm{p}=0,692)$ e a idade $(\mathrm{p}=0,814)$ também não se associaram ao grau de deslizamento (Tabela 3).

Analisando a evolução angular do deslizamento ocorrido, comparando os dois momentos de observação radiográfica (inicial em outro serviço e imediatamente pré-operatória no serviço), observa-se que há diferença estatisticamente significante entre essas medidas $(<0,001)$, indicando aumento do escorregamento (Tabela 4).

Entre os quadris avaliados neste estudo, dois foram submetidos à osteotomia em dois planos, posteriormente à fixação in situ para estabilização e fusão fisária. Um quadril foi submetido à osteotomia trapezoidal quando da chegada no serviço, devido à gravidade do escorregamento que se instalara pela progressão da doença. Uma das pacientes estava já com a fise fechada quando deu entrada no serviço, porém a revisão das radiografias iniciais demonstrou que, no diagnóstico inicial, a fise encontrava-se ainda aberta e, por não ter sido tratada, sofrera progressão do escorregamento. Dos nove quadris restantes, contralaterais dos casos unilaterais, nenhum tinha alterações fisárias, porém, todos foram fixados profilaticamente pela baixa idade dos pacientes. Dentre as possíveis complicações da doença, encontramos um caso de condrólise após osteotomia em dois planos e não observamos necrose avascular nos casos avaliados.

\section{DISCUSSÃO}

Ainda hoje são inúmeras as situações em que o correto diagnóstico do EEPF deixa de ser feito na consulta inicial. O desconhecimento de que, em muitas afecções do quadril, a dor irradia-se para a região interna da coxa e joelho, é apenas uma dessas causas. Alie-se a isso o fato de que, por incidir em jovens adolescentes e préadolescentes, muitas vezes obesos ou com sobrepeso, o médico interpreta a dor e a claudicação como sintomas decorrentes de contusões ou mesmo de sobrecarga na prática de esportes. Assim, são administrados medicamentos para aliviar os sintomas, preconiza-se repouso e, não raramente, solicitam-se exames radiográficos dos joelhos e coluna vertebral, adotando-se condutas outras que retardam o diagnóstico e a instalação de adequada terapêutica.

Waldenström $^{(7)}$ descreveu as etapas fisiopatológicas do processo do EEFE e advogou o tratamento conservador com tração (ou repouso) seguido de imobilização gessada. Diante dos meios radiográficos e do precário arsenal para fixação cirúrgica segundo os padrões da época, além da anestesia pouco segura, a metódica foi aceita e muito praticada na primeira metade do século passado.

Em 1990, Betz et $a l^{(3)}$, em uma publicação isolada e na contramão das tendências recentes pela fixação in situ após diagnóstico, reeditou o tratamento conservador, indicando tração e imobilização gessada do membro afetado. Apesar das constatações de índices aumentados de condrólise e da ineficiência quanto à obtenção da fusão fisária em muitos casos, e a despeito do longo tempo de imobilização exigido, a conduta conquistou adeptos. 
Tabela 1 - Distribuição dos quadris quanto ao sexo, escorregamento inicial, ulterior, número de dias decorridos até o tratamento e idade dos pacientes (dezembro de 1996 a agosto de 2006).

\begin{tabular}{|c|c|c|c|c|c|c|}
\hline Paciente & Sexo & Idade (meses) & Grau inicial & Grau pré-operatório & $\begin{array}{c}\text { Deslizamento } \\
\text { durante observação }\end{array}$ & Tempo (dias) \\
\hline 1 & $\mathrm{~F}$ & 145 & $20^{\circ}$ & $30^{\circ}$ & $10^{\circ}$ & 26 \\
\hline 2 & $M$ & 143 & $51^{\circ}$ & $75^{\circ}$ & $24^{\circ}$ & 28 \\
\hline 3 & $M$ & 143 & $52^{\circ}$ & $71^{\circ}$ & $19^{\circ}$ & 28 \\
\hline 4 & M & 157 & $18^{\circ}$ & $20^{\circ}$ & $2^{\circ}$ & 33 \\
\hline 5 & $\mathrm{~F}$ & 152 & $15^{\circ}$ & $21^{\circ}$ & $6^{\circ}$ & 33 \\
\hline 6 & M & 134 & $28^{\circ}$ & $31^{\circ}$ & $3^{\circ}$ & 36 \\
\hline 7 & M & 118 & $16^{\circ}$ & $16^{\circ}$ & $0^{\circ}$ & 36 \\
\hline 8 & M & 118 & $21^{\circ}$ & $30^{\circ}$ & $9^{\circ}$ & 36 \\
\hline 9 & $M$ & 177 & $36^{\circ}$ & $36^{\circ}$ & $0^{\circ}$ & 37 \\
\hline 10 & M & 177 & $30^{\circ}$ & $30^{\circ}$ & $0^{\circ}$ & 37 \\
\hline 11 & $M$ & 163 & $77^{\circ}$ & $84^{\circ}$ & $7^{\circ}$ & 41 \\
\hline 12 & $M$ & 188 & $30^{\circ}$ & $70^{\circ}$ & $40^{\circ}$ & 47 \\
\hline 13 & $M$ & 188 & $20^{\circ}$ & $29^{\circ}$ & $9^{\circ}$ & 47 \\
\hline 14 & $\mathrm{~F}$ & 143 & $21^{\circ}$ & $22^{\circ}$ & $1^{\circ}$ & 48 \\
\hline 15 & $\mathrm{~F}$ & 143 & $25^{\circ}$ & $35^{\circ}$ & $10^{\circ}$ & 48 \\
\hline 16 & $M$ & 176 & $79^{\circ}$ & $90^{\circ}$ & $11^{\circ}$ & 48 \\
\hline 17 & $\mathrm{~F}$ & 155 & $20^{\circ}$ & $20^{\circ}$ & $0^{\circ}$ & 57 \\
\hline 18 & $F$ & 155 & $30^{\circ}$ & $30^{\circ}$ & $0^{\circ}$ & 57 \\
\hline 19 & $M$ & 163 & $49^{\circ}$ & $52^{\circ}$ & $3^{\circ}$ & 64 \\
\hline 20 & $M$ & 163 & $10^{\circ}$ & $10^{\circ}$ & $0^{\circ}$ & 64 \\
\hline 21 & $F$ & 133 & $42^{\circ}$ & $70^{\circ}$ & $28^{\circ}$ & 76 \\
\hline 22 & $M$ & 175 & $38^{\circ}$ & $41^{\circ}$ & $3^{\circ}$ & 87 \\
\hline 23 & $\mathrm{~F}$ & 131 & $22^{\circ}$ & $29^{\circ}$ & $7^{\circ}$ & 100 \\
\hline 24 & $\mathrm{~F}$ & 131 & $11^{\circ}$ & $11^{\circ}$ & $0^{\circ}$ & 100 \\
\hline 25 & $\mathrm{~F}$ & 136 & $28^{\circ}$ & $35^{\circ}$ & $7^{\circ}$ & 128 \\
\hline 26 & $F$ & 153 & $6^{\circ}$ & $39^{\circ}$ & $33^{\circ}$ & 402 \\
\hline
\end{tabular}

Tabela 2 - Relação entre as variáveis: grau de deslizamento, tempo e idade com relação ao sexo do paciente, segundo aplicação do teste de Mann-Whitney.

\begin{tabular}{c|c|c|c|c|c}
\hline \multirow{2}{*}{ Variável } & Sexo & $\mathrm{n}$ & Média & $\begin{array}{r}\text { Desvio } \\
\text { padrão }\end{array}$ & $\mathrm{p}$ \\
\hline \multirow{2}{*}{ Deslizamento } & $\mathrm{F}$ & 11 & 9,27 & 11,23 & \multirow{2}{*}{0,896} \\
\cline { 2 - 5 } & $\mathrm{M}$ & 15 & 8,67 & 11,25 & \\
\hline \multirow{2}{*}{ Tempo } & $\mathrm{F}$ & 11 & 97,73 & 105,63 & \multirow{2}{*}{0,033} \\
\cline { 2 - 5 } & $\mathrm{M}$ & 15 & 44,60 & 16,06 & \\
\hline \multirow{2}{*}{ Idade } & $\mathrm{F}$ & 11 & 143,36 & 9,53 & \multirow{2}{*}{0,035} \\
\cline { 2 - 5 } & $\mathrm{M}$ & 15 & 158,87 & 23,03 & \\
\hline
\end{tabular}

Pouco antes, Ordeberg et al ${ }^{(5)}$ já haviam alertado para o risco de progressão do escorregamento e conseguinte piora do prognóstico nos casos tratados conservadoramente quando a fise encontrava-se aberta. Carney
Tabela 3 - Aplicação da análise de correlação de Spearman, relacionando tempo e idade e o deslizamento.

\begin{tabular}{c|c|c|c}
\hline Variável & Estatística & Tempo & Idade \\
\hline Deslizamento & $\begin{array}{c}\text { Coeficiente de } \\
\text { correlação }\end{array}$ & $-0,082$ & $-0,048$ \\
\hline & $\begin{array}{c}\text { Significância } \\
(\mathrm{p})\end{array}$ & 0,692 & 0,814 \\
\hline & $\mathrm{n}$ & 26 & 26 \\
\hline
\end{tabular}

Tabela 4 - Aplicação do teste dos postos sinalizados de Wilcoxon entre os valores dos graus iniciais e pré-operatórios.

\begin{tabular}{c|c|c|c|c}
\hline $\begin{array}{c}\text { Par de } \\
\text { variáveis }\end{array}$ & Média & $\mathrm{n}$ & Desvio padrão & $\mathrm{p}$ \\
\hline Grau I & 30,58 & 26 & 18,54 & $<0,001$ \\
\hline Grau pré & 39,50 & 26 & 22,94 & \\
\hline
\end{tabular}



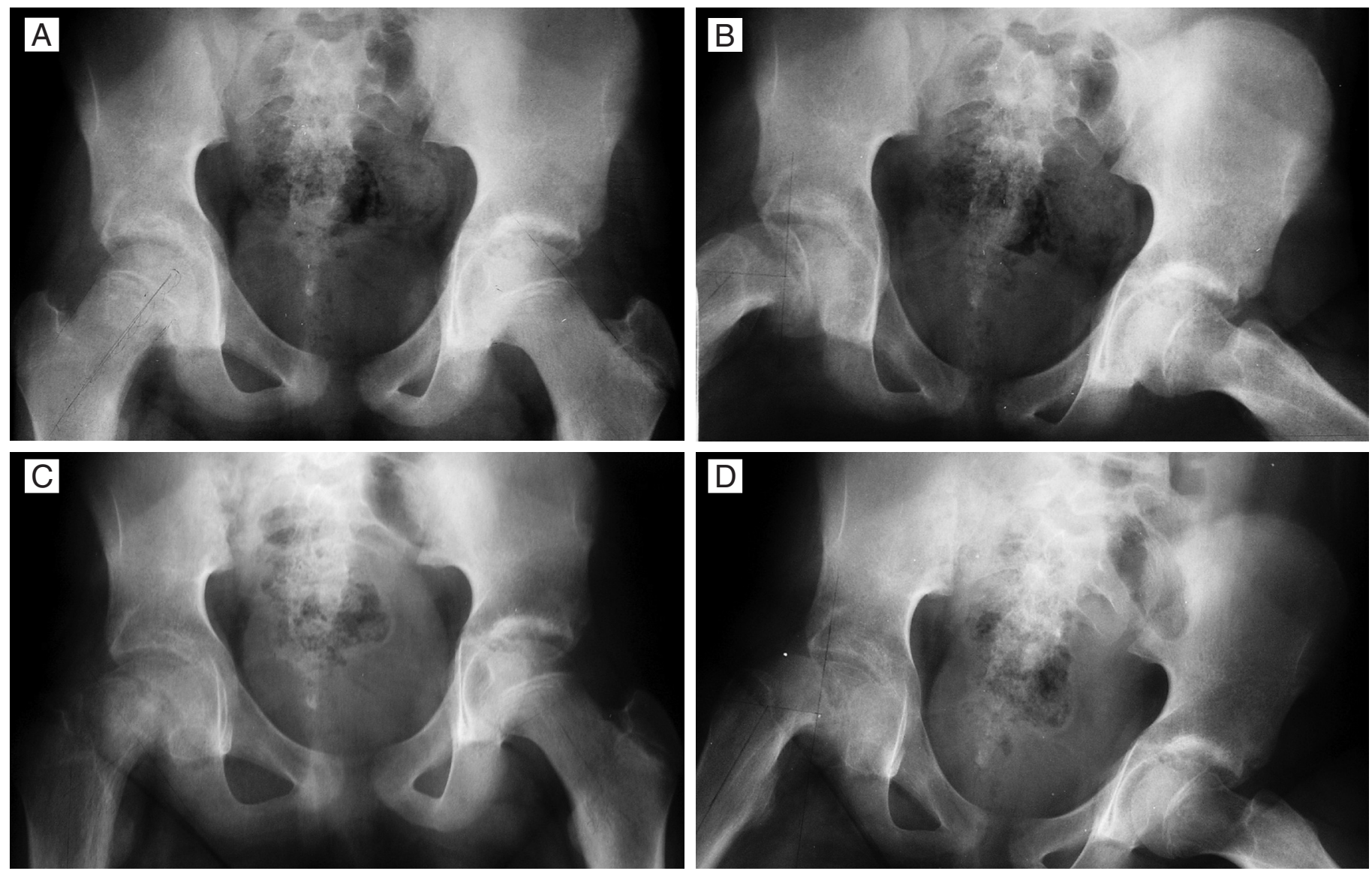

Figura 1 - Caso 21: Paciente do sexo feminino, 11 anos de idade. Radiografias no momento do diagnóstico inicial (A e B), Progressão do escorregamento após 76 dias (C e D).

et $a l^{(4)}$, avaliando a evolução da EEFE tratada de forma conservadora, constataram piora dos graus de deslizamento em $17 \%$ dos pacientes. Observaram, ainda, que $8 \%$ dos casos leves, $18 \%$ nos moderados e $28 \%$ nos casos graves evoluíram para condrólise. Em relação à necrose, os resultados haviam sido igualmente desastrosos, com $2 \%$ nos casos leves e $20 \%$ nos moderados e graves. Ingram et al ${ }^{(8)}$ encontraram $28,9 \%$ de condrólise em 329 casos avaliados, e observaram que essa complicação era maior em pacientes do sexo feminino nos deslizamentos agudos e naqueles pacientes com maiores graus de deslizamento.

Por outro lado, altas taxas de complicações no tratamento cirúrgico ${ }^{(9,10)}$ advinham de épocas em que as técnicas cirúrgicas utilizadas, assim como os materiais de síntese disponíveis não auxiliavam o cirurgião, comprometendo os resultados desse tipo de tratamento. Com o advento dos equipamentos fluoroscópicos atuais, dispositivos para o posicionamento do paciente e melhor conhecimento tático e técnico do cirurgião, a fixação com parafuso único e bem posicionado fez com que as elevadas incidências de complicações baixassem dos níveis referidos de até $36 \%$ para $4,6 \%$ ou $\operatorname{menos}^{(9-13)}$.
Tal fixação in situ pode ser realizada de forma percutânea em casos leves e moderados, com um único parafuso, sem prejuízo para o resultado final. Além da pequena cicatriz cirúrgica, o sangramento é mínimo. Segundo Loder et $a l^{(14)}$, as vantagens da fixação com parafuso único incluem: baixa lesão de partes moles, a fixação evita a progressão do escorregamento e baixa incidência de necrose e condrólise. Excelentes resultados também foram demonstrados por Aronson e Carlson ${ }^{(11)}$ e Blanco et al ${ }^{(13)}$, quando do uso da técnica de fixação com parafuso único em escorregamentos leves e moderados, obtendo de $91 \%$ a $95 \%$ de sucesso.

Os resultados epidemiológicos deste estudo demonstraram que, nas meninas, a média de idade no acometimento foi menor que nos meninos, apresentando diferença estatisticamente significante (Tabela 2). Esses dados reforçam fato já conhecido, uma vez que a doença está relacionada à maturidade óssea, que é mais precoce nas meninas do que meninos. Já a idade no início e o tempo decorrido não conseguem explicar o comportamento do escorregamento subsequente ocorrido. Sabese que a idade cronológica nem sempre corresponde à idade óssea. Além disso, nem todos os pacientes podem 
ter seguido a orientação do tratamento conservador com repouso, fato que altera a velocidade e a gravidade do escorregamento. O caráter progressivo do deslizamento submetido a tratamento conservador foi flagrantemente demonstrado neste estudo pelos valores encontrados nos ângulos das radiografias pré-operatórias, maiores do que os encontrados inicialmente, comprovando a ineficiência do tratamento "conservador" instituído.

Apesar de a quantidade de quadris submetidos ao tratamento conservador identificados neste estudo em 10 anos parecer pouco expressiva $(n=26)$, deve-se enfatizar que, na realidade, o número de pacientes nessa condição deve ser ainda maior. Afinal, dentre os critérios de inclusão para esta pesquisa, consideramos a necessidade de radiografias prévias à chegada no serviço, comprobatórias da doença mediante as quais o médico assistente havia orientado umas das formas de tratamento paliativo (como anti-inflamatórios, analgésicos, imobilização, retirada da carga, repouso ou até mesmo fisioterapia). Muitos dos casos de EEFE admitidos no serviço não possuíam essas radiografias prévias, apesar de relatos em muitos prontuários de que o paciente havia sido atendido anteriormente e que fora radiografado. Os pacientes, portanto, nem sempre trazem seus exames prévios, o que dificulta a realização do estudo.

Além desses pacientes que não portavam as radiografias prévias, tivemos também casos lamentáveis que não haviam sido sequer examinados e muito menos radiografados em outros serviços, apesar do quadro típico de EEPF, e ainda casos em que foram radiografados somente os joelhos ou até mesmo a bacia, mas nos quais o diagnóstico não fora realizado. Se incluíssemos esses casos no estudo, somaríamos pelo menos 35 pacientes, resultando em percentual de $8,7 \%$ de todos os prontuários avaliados.

Deve-se acrescentar a esta discussão o fato de que, recentemente, o impacto acetabular e suas consequências, como a lesão labral e a evolução osteoartrósica, têm sido associadas aos EEPF em todos os graus, conforme relatos de Leunig et al ${ }^{(15)}$ e Ganz et $a l^{(16)}$. Esses autores descreveram a relação direta entre o EEPF e o impacto acetabular decorrente de um processo insuficiente na remodelação do colo femoral. Desta forma, a conduta expectante após o correto diagnóstico de EEPF não se justifica nos dias de hoje, fato que nos preocupa como especialistas que, muitas vezes, somos procurados quando a gravidade do escorregamento já é de tal forma acentuada, que dificulta muito a possibilidade de uma terapêutica isenta de complicações.

\section{CONCLUSÃO}

O tratamento conservador não é eficaz para evitar o agravamento do escorregamento no EEPF. As complicações advindas desta postura terapêutica de observação são aquelas conhecidas com a evolução natural de uma doença progressiva, ou seja, diferentes graus de alteração biomecânica do quadril e suas prováveis repercussões a médio e longo prazos.

\section{REFERÊNCIAS}

1. Santili C. Epifisiólise. Rev Bras Ortop. 2001;36(3):49-56.

2. Loder RT, Wittenberg B, DeSilva G. Slipped capital femoral epiphysis associated with endocrine disorders. J Pediatr Orthop. 1995;15(3):349-56.

3. Betz RR, Steel HH, Emper WD, Huss GK, Clancy M. Treatment of slipped capital femoral epiphysis. Spica-cast immobilization. J Bone Joint Surg Am. 1990;72(4):587-600.

4. Carney BT, Weinstein SL, Noble J. Long-term follow-up of slipped capital femoral epiphysis. J Bone Joint Surg Am. 1991;73(5):667-74.

5. Ordeberg G, Hansson LI, Sandström S. Slipped capital femoral epiphysis in southern Sweden. Long-term result with no treatment or symptomatic primary treatment. Clin Orthop Relat Res. 1984;(191):95-104.

6. Southwick WO. Osteotomy through the lesser trochanter for slipped capital femoral epiphysis. J Bone Joint Surg Am. 1967;49(5):807-35.

7. Waldenström H. On necrosis of the joint cartilage by epiphyseolysis capitis femoris. Acta Chir Scand. 1930;67:936-46.

8. Ingram AJ, Clarke MS, Clark CS Jr, Marshall WR. Chondrolysis complicating slipped capital femoral epiphysis. Clin Orthop Relat Res. 1982;(165):99-109.

9. Walters R, Simon SR. Joint destruction: a sequel of unrecognized pin penetration in patients with slipped capital femoral epiphyses. In: The Hip: Proceedings

of the eighth open scientific meeting of the Hip Society. Philadelphia: C.V. Mosby Company; 1980.

10. Riley PM, Weiner DS, Gillespie R, Weiner SD. Hazards of internal fixation in the treatment of slipped capital femoral epiphysis. J Bone Joint Surg Am. 1990;72(10):1500-9.

11. Aronson DD, Carlson WE. Slipped capital femoral epiphysis. A prospective study of fixation with a single screw. J Bone Joint Surg Am. 1992;74(6):810-9.

12. Morrissy RT. Principles of in situ fixation in chronic slipped capital femoral epiphysis. Instr Course Lect. 1989;38:257-62.

13. Blanco JS, Taylor B, Johnston CE. Comparison of single pin versus multiple pin fixation in treatment of slipped capital femoral epiphysis. J Pediatr Orthop. 1992;12(3):384-9.

14. Loder RT, Aronsson DD, Dobbs MB, Weinstein SL. Slipped capital femoral epiphysis. J Bone Joint Surg Am. 2000;82:1170.

15. Leunig M, Casillas MM, Hamlet M, Hersche O, Nötzli H, Slongo T, et al. Sliped capital femoral epiphysis: early mechanical damage to the acetabular cartilage by a prominent femoral metaphysic. Acta Orthop Scand. 2000;71(4):370-5.

16. Ganz R, Parvizi J, Beck M, Leunig M, Nötzli H, Siebenrock KA Femoroacetabular impingement: a cause for osteoarthritis of the hip. Clin Orthop Relat Res. 2003;(417):112-20. 